\title{
Comparison of Menstruation-Related Symptoms Before and During Menstruation of University Students in Japan, a Year after the COVID-19 Pandemic
}

\author{
Yukie Matsuura ${ }^{1}$, Nam Hoang $\operatorname{Tran}^{2} \&$ Toshiyuki Yasui ${ }^{1}$ \\ ${ }^{1}$ Department of Reproductive and Menopausal Medicine, Graduate School of Biomedical Sciences, Tokushima \\ University, Tokushima, Japan \\ ${ }^{2}$ Research Center for Higher Education, Tokushima University, Tokushima, Japan \\ Correspondence: Yukie Matsuura, Department of Reproductive and Menopausal Medicine, Graduate School of \\ Biomedical Sciences, Tokushima University, Tokushima 770-8503, Japan. Tel: +81-88-633-7628. E-mail: \\ y.matsuura@tokushima-u.ac.jp
}

Received: January 29, 2022 Accepted: February 21, 2022 Online Published: February 23, 2022

doi:10.5539/gjhs.v14n4p1

URL: https://doi.org/10.5539/gjhs.v14n4p1

\begin{abstract}
The coronavirus disease 2019 (COVID-19) pandemic affected the daily lifestyle of people, including many aspects affecting young women. Subsequent to the COVID-19 pandemic stress and anxiety have been reported related to menstrual disorders (Takmaz, Gundogmus, Okten, \& Gunduz, 2021). The purpose of this study was to investigate the intensity and to compare menstruation-related symptoms before and during menstruation among university students in Japan. We conducted an online cross-sectional study from May to July 2021 using a menstrual distress questionnaire (MDQ) to assess symptoms experienced before and during menstruation. Our results showed that of 141 students, five students (3.5\%) did not report any symptoms before menstruation and one student $(0.7 \%)$ had no symptoms during menstruation. We found that the most frequently experienced symptoms before menstruation were skin blemishes or disorder, mood swings, irritability, swelling, cramps, fatigue, take naps, stay in bed, feeling sad or blue, weight gain and difficulty concentrating. The most frequently experienced symptoms during menstruation were cramps, fatigue, irritability, mood swings, take naps, stay in bed, feeling sad or blue, backache, swelling, skin blemish or disorder, and poor school/work performance. The total MDQ score was significantly higher during menstruation than before menstruation. Among the MDQ eight scales, the scores of five scales including pain, autonomic reaction, impaired concentration, behavior change, and control were significantly higher during menstruation than before menstruation. The prevalence of increased appetite and craving for sweets were higher than MDQ symptoms before menstruation. The prolonged exposure to pandemic may have more effect on psychological symptoms than on physical symptoms.
\end{abstract}

Keywords: COVID-19 pandemic, menstruation-related symptoms, menstrual distress questionnaire, young women

\section{Introduction}

Since the declaration of the coronavirus disease 2019 (COVID-19) pandemic by the World Health Organization (WHO) on March 11, 2020, the lifestyle of people has undergone many changes. The COVID-19 pandemic has influenced students' lifestyle as well as their physical and mental health. For university students, various changes in their academic life occurred, such as restrictions in access to campus and increased online classes. A prospective cohort study conducted before and during the COVID-19 lockdown amongst university students in Saudi Arabia showed that physical activity had decreased, sedentary time had increased, and that although their level of sleeping had decreased but sleeping time had increased (Jalal, Beth, Al-Hassan, \& Alshealah, 2021). A cross-sectional survey amongst medical students in nine countries including Japan reported that $90 \%$ of respondents mentioned a transition to online education, and $96 \%$ of students increased their time spent in front of screens such as computers, cell phones, tablets, and others electronic devices (Perez-Dominguez et al., 2021). The same study also reported that about a half of the students reported negative changes in their studying, sleeping and eating habits, and they frequently experienced the onset and increasing mental and physical health symptoms such as backache, asthenopia, irritability, and emotional instability (Perez-Dominguez et al., 2021). 
There were some reports regarding the association of the COVID-19 pandemic with menstruation and menstruation-related symptoms including premenstrual syndrome (PMS), premenstrual dysphoric disorder (PMDD) and dysmenorrhea, which affected the daily and academic life of young women, such as being absent from school (Armour et al., 2019; Tadakawa, Takeda, Monma, Koga, \& Yaegashi, 2016) or by the negative effects on their concentration (Armour et al., 2019). It was reported that for women in Turkey, duration of menstruation decreased compared to their experiences before the COVID-19 outbreak and the severity of anxiety and stress positively correlated with the degree of menstrual and somatic symptoms (using the menstruation symptom questionnaire -MSQ) (Demir, Sal, \& Comba, 2021). It was reported that $53 \%$ of women in Ireland had worse premenstrual symptoms and 30\% women had new dysmenorrhea after the pandemic breakout (Phelan, Behan, \& Owens, 2021). Moreover, the number of women having mental health symptoms increased, and the women with mental health symptoms were also more likely to report painful periods and worsening premenstrual symptoms (Phelan et al., 2021). It was further reported that female healthcare workers in Turkey had an association between the COVID-19 pandemic-induced anxiety, perceived stress, depressive symptoms, and increased prevalence of menstrual cycle irregularity (Takmaz, Gundogmus, Okten, \& Gunduz, 2021). A longitudinal study in Brazil before and during pandemic also showed that the total premenstrual screening tool score showed no change in the presence and severity of premenstrual symptoms between the two periods, but that the "anxiety/stress" symptoms of this tool revealed that it was more severe in the students before the pandemic (Freitas, de Medeiros, \& Lopes, 2021). Thus, stress and anxiety may be related to menstrual abnormality during the pandemic. Since more than a year of the COVID-19 pandemic has passed, students, however, may be getting used to coexisting with COVID-19 with less anxiety. Although menstrual cycle-related symptoms include physical, behavioral, and emotional symptoms (Clayton, 2008), to our knowledge, comparison of menstruation-related symptom in young women between before and during menstruation have not been reported.

This study therefore aimed to investigate the intensity of menstruation-related symptoms before and during menstruation and to compare menstrual symptoms before and during menstruation in Japanese university students, a year after the start of the COVID-19 pandemic.

\section{Materials and Methods}

\subsection{Participants}

We recruited young female university students who were enrolled in the health science department of a university in Japan. For the purpose of recruitment, we announced the recruitment during online classes or face-to-face classes with the 320 students. After these sessions, 169 students agreed to participate voluntarily.

\subsection{Data Collection Procedure}

Data collection for this study was conducted between May to July 2021.The questionnaire for survey was created by the authors, then converted into the online form by using SurveyMonkey (https://jp.surveymonkey.com/).

The researchers provided an explanation relating to the purpose of the survey and the protocol and then asked students to participate in the survey. The study was anonymous and completely voluntary. All questions were compulsory, but it was possible to quit the questionnaire at any time. Students could access the online survey by clicking on the URL or scanning the QR code provided. Students needed to check a button for informed consent before answering the survey questions; they could continue the survey once they had provided informed consent, or if not they could end their participation. The study was approved by the Ethics Committee of Tokushima University Hospital (approval number: 3932).

\subsection{Measurements}

\subsubsection{Demographic Characteristics}

For this study, we developed a questionnaire consisting of demographic information including age, body weight, body height, age of menarche, currently visiting gynecological doctors and taking hormone therapy, present menstrual conditions (menstrual cycle length, regularity, duration of menstrual bleeding and perceived amount of menstrual bleeding). Body Mass Index (BMI) was calculated as the body weight $(\mathrm{kg}) /$ body height $\left(\mathrm{m}^{2}\right)$.

\subsubsection{Menstruation-Related Measurements}

This part of the questionnaire consisted of questions on information about the intensity of menstruation-related symptoms. The intensities of menstruation-related symptoms were assessed using the menstrual distress questionnaire (MDQ). The MDQ which has been a standard method widely used for measuring menstruation-related symptoms (Moos, 1968) contains 46 symptom items grouped into eight scales. Among the eight scales, three somatic scales are for pain, water retention, and autonomic reactions, another three scales are for 
mood and behavioral changes, specifically negative affect, impaired concentration and behavior change. The last two scales are for arousal and control (Moos, 2010). Students were asked to rate their perceived intensity of each item during three menstrual phases including before menstruation (4 days before mensuration), during menstruation and the remainder of the cycle. For each item, the participants could rate their experiences on a five-point scale from 0 to 4 , where 0 was no experience of symptom, 1 was present and mild, 2 was present and moderate, 3 was present and strong and 4 was present and severe. The Cronbach's alpha coefficient in this study was 0.84 and 0.86 for total MDQ score before and during menstruation, respectively. We also investigated three eating habit symptoms, comprising an increase in appetite, craving for sweets and snacks, which we found specific change before and during menstruation (Matsuura, Inoue, Kidani, \& Yasui, 2020). Each symptom was also rated on a five-point scale.

\subsection{Statistics Analysis}

We performed the statistical analysis using SPSS version 28.0 for Windows (IBM Corp., Armonk, NY, USA). Categorical variables were presented as whole numbers with percentages or means with standard deviations. The MDQ score was calculated by total score and score for each of the eight scales, presented as median (25th percentile, 75 th percentile). The three eating habit symptoms were also presented in the same way. We used the Wilcoxon signed rank test to compare the intensity of MDQ total score, each of the eight scales' score and three eating habit symptoms' score before and during menstruation. All p-values $<0.05$ were regarded as statistically significant.

\section{Results}

We recruited from 320 female students for the survey, of whom 169 students participated in the study. We excluded 28 students, comprising 18 students who did not complete the MDQ items and 10 students who were under hormonal therapy for their gynecological diseases. Subsequently, we used the data of 141 students for analysis.

\subsection{Demographic Characteristics}

The mean age of the students was $19.8 \pm 3.1$ years, and the mean menarche age was $12.1 \pm 1.4$ years. Among them, $87.9 \%$ of students reported that their menstrual cycle length ranged from 25 to 38 days. Menstrual duration for most of the students ranged from 3 to 7 days. The amount of menstrual bleeding was perceived as moderate by $75.9 \%$ of the students (Table 1).

Table 1. Demographic characteristics $(\mathrm{n}=141)$

\begin{tabular}{|c|c|c|c|}
\hline Characteristic & Category & $\mathrm{n}$ & $\%$ \\
\hline Age (years) ${ }^{\mathrm{a}}$ & & $19.8(3.1)$ & \\
\hline Height $(\mathrm{cm})^{\mathrm{a}}$ & & $157.4(4.9)$ & \\
\hline Weight $(\mathrm{kg})^{\mathrm{a}}$ & & $51.4(6.2)$ & \\
\hline Menarche age (years) ${ }^{a}$ & & $12.1(1.4)$ & \\
\hline Body mass index $\left(\mathrm{kg} / \mathrm{m}^{2}\right)^{\mathrm{a}}$ & & $20.7(2.1)$ & \\
\hline \multirow[t]{4}{*}{ Menstrual cycle length } & 24 days or less & 2 & 1.4 \\
\hline & 25-38 days & 124 & 87.9 \\
\hline & 39 days or more & 14 & 9.9 \\
\hline & No menstruation for more than 3 months & 1 & 0.7 \\
\hline \multirow[t]{3}{*}{ Menstrual cycle regularity } & Regular & 67 & 47.5 \\
\hline & Sometimes irregular & 65 & 46.1 \\
\hline & Irregular & 9 & 6.4 \\
\hline \multirow[t]{2}{*}{ Duration of menstruation } & 3-7 days & 137 & 97.2 \\
\hline & 8 days or more & 4 & 2.8 \\
\hline \multirow[t]{3}{*}{ Perceived amount of menstrual bleeding } & Light & 12 & 8.5 \\
\hline & Moderate & 107 & 75.9 \\
\hline & Heavy & 22 & 15.6 \\
\hline
\end{tabular}

${ }^{\mathrm{a}}$ Mean (standard deviation). 


\subsection{Prevalence of $M D Q$ Symptoms}

Five students $(3.5 \%)$ did not report any symptoms before menstruation and one student $(0.7 \%)$ similarly during menstruation. The range of the MDQ total score before menstruation was 0-109 points and the range of the score during menstruation was $0-124$ points.

Regarding symptoms before menstruation, the most frequently experienced symptoms were skin blemish or disorder (68.8\%), mood swings (68.1\%), irritability (63.8\%), swelling (62.4\%), cramps (61.0\%), fatigue, take naps, stay in bed, feeling sad or blue, weight gain and difficulty concentrating (Table 2).

Table 2. Prevalence of MDQ symptoms before menstruation by the level of intensity ( $n=141)$

\begin{tabular}{|c|c|c|c|c|c|c|c|c|c|c|c|}
\hline \multirow{3}{*}{ Scales } & \multirow{3}{*}{ Symptoms } & \multicolumn{10}{|c|}{ Intensity } \\
\hline & & \multicolumn{2}{|c|}{ None } & \multicolumn{2}{|c|}{ Mild } & \multicolumn{2}{|c|}{ Moderate } & \multicolumn{2}{|c|}{ Strong } & \multicolumn{2}{|c|}{ Severe } \\
\hline & & $\mathrm{n}$ & $\%$ & $\mathrm{n}$ & $\%$ & $\mathrm{n}$ & $\%$ & $\mathrm{n}$ & $\%$ & $\mathrm{n}$ & $\%$ \\
\hline \multirow{6}{*}{ Pain } & Muscle stiffness & 120 & 85.1 & 15 & 10.6 & 3 & 2.1 & 2 & 1.4 & 1 & 0.7 \\
\hline & Headache & 88 & 62.4 & 27 & 19.1 & 18 & 12.8 & 3 & 2.1 & 5 & 3.5 \\
\hline & Cramps & 55 & 39.0 & 43 & 30.5 & 28 & 19.9 & 14 & 9.9 & 1 & 0.7 \\
\hline & Backache & 80 & 56.7 & 33 & 23.4 & 23 & 16.3 & 3 & 2.1 & 2 & 1.4 \\
\hline & Fatigue & 58 & 41.1 & 27 & 19.1 & 33 & 23.4 & 18 & 12.8 & 5 & 3.5 \\
\hline & General aches and pains & 102 & 72.3 & 24 & 17.0 & 10 & 7.1 & 5 & 3.5 & 0 & 0.0 \\
\hline \multirow{4}{*}{ Water retention } & Weight gain & 75 & 53.2 & 31 & 22.0 & 17 & 12.1 & 15 & 10.6 & 3 & 2.1 \\
\hline & Skin blemish or disorder & 44 & 31.2 & 32 & 22.7 & 33 & 23.4 & 25 & 17.7 & 7 & 5.0 \\
\hline & Painful or tender breasts & 81 & 57.4 & 22 & 15.6 & 20 & 14.2 & 15 & 10.6 & 3 & 2.1 \\
\hline & Swelling (breasts abdomen) & 53 & 37.6 & 31 & 22.0 & 37 & 26.2 & 17 & 12.1 & 3 & 2.1 \\
\hline \multirow{4}{*}{ Autonomic reactions } & Dizziness, faintness & 89 & 63.1 & 28 & 19.9 & 16 & 11.3 & 7 & 5.0 & 1 & 0.7 \\
\hline & Cold sweats & 130 & 92.2 & 7 & 5.0 & 3 & 2.1 & 1 & 0.7 & 0 & 0.0 \\
\hline & Nausea, vomiting & 124 & 87.9 & 7 & 5.0 & 7 & 5.0 & 2 & 1.4 & 1 & 0.7 \\
\hline & Hot flashes & 106 & 75.2 & 15 & 10.6 & 16 & 11.3 & 2 & 1.4 & 2 & 1.4 \\
\hline \multirow{8}{*}{ Negative affect } & Loneliness & 93 & 66.0 & 14 & 9.9 & 21 & 14.9 & 6 & 4.3 & 7 & 5.0 \\
\hline & Anxiety & 88 & 62.4 & 23 & 16.3 & 18 & 12.8 & 5 & 3.5 & 7 & 5.0 \\
\hline & Mood swings & 45 & 31.9 & 30 & 21.3 & 27 & 19.1 & 26 & 18.4 & 13 & 9.2 \\
\hline & Crying & 102 & 72.3 & 15 & 10.6 & 8 & 5.7 & 9 & 6.4 & 7 & 5.0 \\
\hline & Irritability & 51 & 36.2 & 27 & 19.1 & 19 & 13.5 & 31 & 22.0 & 13 & 9.2 \\
\hline & Tension & 102 & 72.3 & 17 & 12.1 & 13 & 9.2 & 7 & 5.0 & 2 & 1.4 \\
\hline & Feeling sad or blue & 63 & 44.7 & 26 & 18.4 & 29 & 20.6 & 12 & 8.5 & 11 & 7.8 \\
\hline & Restlessness & 101 & 71.6 & 21 & 14.9 & 14 & 9.9 & 2 & 1.4 & 3 & 2.1 \\
\hline \multirow{8}{*}{ Impaired Concentration } & Insomnia & 111 & 78.7 & 12 & 8.5 & 15 & 10.6 & 2 & 1.4 & 1 & 0.7 \\
\hline & Forgetfulness & 124 & 87.9 & 7 & 5.0 & 9 & 6.4 & 0 & 0.0 & 1 & 0.7 \\
\hline & Confusion & 110 & 78.0 & 16 & 11.3 & 10 & 7.1 & 2 & 1.4 & 3 & 2.1 \\
\hline & Poor judgment & 111 & 78.7 & 15 & 10.6 & 12 & 8.5 & 2 & 1.4 & 1 & 0.7 \\
\hline & Difficulty concentrating & 78 & 55.3 & 28 & 19.9 & 23 & 16.3 & 9 & 6.4 & 3 & 2.1 \\
\hline & Distractible & 83 & 58.9 & 27 & 19.1 & 20 & 14.2 & 8 & 5.7 & 3 & 2.1 \\
\hline & Minor Accidents & 98 & 69.5 & 21 & 14.9 & 14 & 9.9 & 7 & 5.0 & 1 & 0.7 \\
\hline & Poor motor coordination & 96 & 68.1 & 26 & 18.4 & 11 & 7.8 & 5 & 3.5 & 3 & 2.1 \\
\hline
\end{tabular}




\begin{tabular}{|c|c|c|c|c|c|c|c|c|c|c|}
\hline \multirow{5}{*}{ Behavior change } & Poor school/work performance & 81 & 57.4 & 3222.7 & 12 & 8.5 & 9 & 6.4 & 7 & 5.0 \\
\hline & Take naps, stay in bed & 62 & 44.0 & 1913.5 & 20 & 14.2 & 23 & 16.3 & 17 & 12.1 \\
\hline & Stay at home & 89 & 63.1 & 1611.3 & 18 & 12.8 & 11 & 7.8 & 7 & 5.0 \\
\hline & Avoid social activities & 93 & 66.0 & 2417.0 & 14 & 9.9 & 9 & 6.4 & 1 & 0.7 \\
\hline & Decreased efficiency & 83 & 58.9 & 2819.9 & 15 & 10.6 & 8 & 5.7 & 7 & 5.0 \\
\hline \multirow{5}{*}{ Arousal } & Affectionate & 88 & 62.4 & 2014.2 & 21 & 14.9 & 6 & 4.3 & 6 & 4.3 \\
\hline & Orderliness & 113 & 80.1 & 128.5 & 14 & 9.9 & 2 & 1.4 & 0 & 0.0 \\
\hline & Excitement & 121 & 85.8 & 139.2 & 4 & 2.8 & 3 & 2.1 & 0 & 0.0 \\
\hline & Feelings of well-being & 92 & 65.2 & 2517.7 & 18 & 12.8 & 5 & 3.5 & 1 & 0.7 \\
\hline & Bursts of energy, activity & 97 & 68.8 & 2920.6 & 11 & 7.8 & 3 & 2.1 & 1 & 0.7 \\
\hline \multirow{6}{*}{ Control } & Feelings of suffocation & 123 & 87.2 & $8 \quad 5.7$ & 8 & 5.7 & 2 & 1.4 & 0 & 0.0 \\
\hline & Chest pains & 123 & 87.2 & 117.8 & 6 & 4.3 & 0 & 0.0 & 1 & 0.7 \\
\hline & Ringing in the ears & 120 & 85.1 & 139.2 & 7 & 5.0 & 1 & 0.7 & 0 & 0.0 \\
\hline & Heart pounding & 125 & 88.7 & 96.4 & 5 & 3.5 & 1 & 0.7 & 1 & 0.7 \\
\hline & Numbness, tingling & 134 & 95.0 & $5 \quad 3.5$ & 1 & 0.7 & 1 & 0.7 & 0 & 0.0 \\
\hline & Blind spots, fuzzy vision & 128 & 90.8 & 96.4 & 4 & 2.8 & 0 & 0.0 & 0 & 0.0 \\
\hline
\end{tabular}

Subsequently, regarding symptoms during menstruation, the most frequently experienced symptoms were cramps (93.6\%), fatigue (77.3\%), irritability (75.9\%), mood swings (73.8\%), take naps, stay in bed (72.3\%), feeling sad or blue, backache, swelling (breasts, abdomen), skin blemish or disorder, and poor school/work performance (Table $3)$.

Table 3. Prevalence of MDQ symptoms during menstruation by the level of intensity ( $\mathrm{n}=141$ )

\begin{tabular}{|c|c|c|c|c|c|c|c|c|c|c|}
\hline \multirow{3}{*}{ Scales } & \multirow{3}{*}{ Symptoms } & \multicolumn{9}{|c|}{ Intensity } \\
\hline & & \multicolumn{2}{|c|}{ None } & Mild & \multicolumn{2}{|c|}{ Moderate } & \multicolumn{2}{|c|}{ Strong } & \multicolumn{2}{|c|}{ Severe } \\
\hline & & $\mathrm{n}$ & $\%$ & $\mathrm{n} \quad \%$ & $\mathrm{n}$ & $\%$ & $\mathrm{n}$ & $\%$ & $\mathrm{n}$ & $\%$ \\
\hline \multirow{6}{*}{ Pain } & Muscle stiffness & 110 & 78.0 & 1510.6 & 9 & 6.4 & 5 & 3.5 & 2 & 1.4 \\
\hline & Headache & 77 & 54.6 & 2316.3 & 30 & 21.3 & 7 & 5.0 & 4 & 2.8 \\
\hline & Cramps & 9 & 6.4 & 2618.4 & 38 & 27.0 & 51 & 36.2 & 17 & 12.1 \\
\hline & Backache & 42 & 29.8 & 2417.0 & 29 & 20.6 & 31 & 22.0 & 15 & 10.6 \\
\hline & Fatigue & 32 & 22.7 & 2517.7 & 37 & 26.2 & 34 & 24.1 & 13 & 9.2 \\
\hline & General aches and pains & 74 & 52.5 & 2819.9 & 27 & 19.1 & 9 & 6.4 & 3 & 2.1 \\
\hline \multirow{4}{*}{ Water retention } & Weight gain & 79 & 56.0 & 2719.1 & 25 & 17.7 & 9 & 6.4 & 1 & 0.7 \\
\hline & Skin blemish or disorder & 45 & 31.9 & 3927.7 & 32 & 22.7 & 19 & 13.5 & 6 & 4.3 \\
\hline & Painful or tender breasts & 75 & 53.2 & 3222.7 & 22 & 15.6 & 9 & 6.4 & 3 & 2.1 \\
\hline & Swelling (breasts abdomen) & 43 & 30.5 & 3827.0 & 39 & 27.7 & 18 & 12.8 & 3 & 2.1 \\
\hline \multirow{4}{*}{ Autonomic reactions } & Dizziness, faintness & 63 & 44.7 & 3122.0 & 35 & 24.8 & 8 & 5.7 & 4 & 2.8 \\
\hline & Cold sweats & 116 & 82.3 & 117.8 & 6 & 4.3 & 7 & 5.0 & 1 & 0.7 \\
\hline & Nausea, vomiting & 107 & 75.9 & 1510.6 & 13 & 9.2 & 3 & 2.1 & 3 & 2.1 \\
\hline & Hot flashes & 98 & 69.5 & 2719.1 & 10 & 7.1 & 5 & 3.5 & 1 & 0.7 \\
\hline
\end{tabular}




\begin{tabular}{|c|c|c|c|c|c|c|c|c|c|c|}
\hline \multirow{8}{*}{ Negative affect } & Loneliness & 87 & 61.7 & 2417.0 & 21 & 14.9 & 7 & 5.0 & 2 & 1.4 \\
\hline & Anxiety & 81 & 57.4 & 3222.7 & 20 & 14.2 & 6 & 4.3 & 2 & 1.4 \\
\hline & Mood swings & 37 & 26.2 & 3726.2 & 35 & 24.8 & 25 & 17.7 & 7 & 5.0 \\
\hline & Crying & 103 & 73.0 & 2014.2 & 7 & 5.0 & 9 & 6.4 & 2 & 1.4 \\
\hline & Irritability & 34 & 24.1 & 4330.5 & 30 & 21.3 & 22 & 15.6 & 12 & 8.5 \\
\hline & Tension & 90 & 63.8 & 2417.0 & 17 & 12.1 & 6 & 4.3 & 4 & 2.8 \\
\hline & Feeling sad or blue & 40 & 28.4 & 3424.1 & 38 & 27.0 & 16 & 11.3 & 13 & 9.2 \\
\hline & Restlessness & 93 & 66.0 & 3021.3 & 11 & 7.8 & 4 & 2.8 & 3 & 2.1 \\
\hline \multirow{8}{*}{ Impaired Concentration } & Insomnia & 104 & 73.8 & 1913.5 & 15 & 10.6 & 2 & 1.4 & 1 & 0.7 \\
\hline & Forgetfulness & 120 & 85.1 & 107.1 & 10 & 7.1 & 0 & 0.0 & 1 & 0.7 \\
\hline & Confusion & 104 & 73.8 & 1913.5 & 17 & 12.1 & 1 & 0.7 & 0 & 0.0 \\
\hline & Poor judgment & 101 & 71.6 & 1611.3 & 19 & 13.5 & 5 & 3.5 & 0 & 0.0 \\
\hline & Difficulty concentrating & 53 & 37.6 & 2719.1 & 37 & 26.2 & 20 & 14.2 & 4 & 2.8 \\
\hline & Distractible & 61 & 43.3 & 3323.4 & 31 & 22.0 & 13 & 9.2 & 3 & 2.1 \\
\hline & Minor Accidents & 87 & 61.7 & 2316.3 & 24 & 17.0 & 5 & 3.5 & 2 & 1.4 \\
\hline & Poor motor coordination & 67 & 47.5 & 2014.2 & 26 & 18.4 & 19 & 13.5 & 9 & 6.4 \\
\hline \multirow{5}{*}{ Behavior change } & Poor school/work performance & 45 & 31.9 & 3625.5 & 34 & 24.1 & 12 & 8.5 & 14 & 9.9 \\
\hline & Take naps, stay in bed & 39 & 27.7 & 2618.4 & 30 & 21.3 & 25 & 17.7 & 21 & 14.9 \\
\hline & Stay at home & 66 & 46.8 & 2014.2 & 22 & 15.6 & 20 & 14.2 & 13 & 9.2 \\
\hline & Avoid social activities & 82 & 58.2 & 2114.9 & 17 & 12.1 & 16 & 11.3 & 5 & 3.5 \\
\hline & Decreased efficiency & 61 & 43.3 & 2719.1 & 30 & 21.3 & 15 & 10.6 & 8 & 5.7 \\
\hline \multirow{5}{*}{ Arousal } & Affectionate & 89 & 63.1 & 2618.4 & 15 & 10.6 & 6 & 4.3 & 5 & 3.5 \\
\hline & Orderliness & 117 & 83.0 & 96.4 & 14 & 9.9 & 1 & 0.7 & 0 & 0.0 \\
\hline & Excitement & 128 & 90.8 & 96.4 & 3 & 2.1 & 1 & 0.7 & 0 & 0.0 \\
\hline & Feelings of well-being & 96 & 68.1 & 2517.7 & 16 & 11.3 & 4 & 2.8 & 0 & 0.0 \\
\hline & Bursts of energy, activity & 113 & 80.1 & 1913.5 & 7 & 5.0 & 2 & 1.4 & 0 & 0.0 \\
\hline \multirow{6}{*}{ Control } & Feelings of suffocation & 123 & 87.2 & 64.3 & 7 & 5.0 & 2 & 1.4 & 3 & 2.1 \\
\hline & Chest pains & 122 & 86.5 & 107.1 & 8 & 5.7 & 0 & 0.0 & 1 & 0.7 \\
\hline & Ringing in the ears & 119 & 84.4 & 1510.6 & 6 & 4.3 & 1 & 0.7 & 0 & 0.0 \\
\hline & Heart pounding & 118 & 83.7 & 139.2 & 7 & 5.0 & 2 & 1.4 & 1 & 0.7 \\
\hline & Numbness, tingling & 135 & 95.7 & 10.7 & 3 & 2.1 & 2 & 1.4 & 0 & 0.0 \\
\hline & Blind spots, fuzzy vision & 126 & 89.4 & 107.1 & 4 & 2.8 & 1 & 0.7 & 0 & 0.0 \\
\hline
\end{tabular}

\subsection{Comparison of MDQ Symptoms' Intensities Before and During Menstruation}

The total MDQ score was significantly higher during menstruation than before menstruation. Among eight scales, the scores of five scales including pain, autonomic reaction, impaired concentration, behavior change, and control were significantly higher during menstruation than before menstruation. In contrast, the score of arousal was significantly higher before menstruation than during menstruation (Table 4). 
Table 4. Comparison of MDQ symptoms' intensities before and during menstruation by subcategory ( $\mathrm{n}=141)$

\begin{tabular}{lllllllll}
\hline Scales & No. of symptoms & \multicolumn{6}{l}{ Before menstruation } & \multicolumn{2}{l}{ During menstruation } & $p$-value \\
\hline Pain & 6 & 3.0 & $(1.0$, & $6.0)$ & 7.0 & $(4.0$, & $11.0)$ & $<0.001$ \\
Water retention & 4 & 3.0 & $(2.0$, & $7.0)$ & 4.0 & $(1.0$, & $6.0)$ & 0.392 \\
Autonomic reaction & 4 & 1.0 & $(0.0$, & $2.0)$ & 1.0 & $(0.0$, & $3.0)$ & $<0.001$ \\
Negative affect & 8 & 5.0 & $(1.0$, & $11.0)$ & 7.0 & $(2.0$, & $12.0)$ & 0.121 \\
Impaired Concentration & 8 & 2.0 & $(0.0$, & $6.0)$ & 4.0 & $(1.0$, & $10.0)$ & $<0.001$ \\
Behavior change & 5 & 2.0 & $(0.0$, & $6.5)$ & 6.0 & $(1.5$, & $10.0)$ & $<0.001$ \\
Arousal & 5 & 1.0 & $(0.0$, & $4.0)$ & 1.0 & $(0.0$, & $3.0)$ & $<0.001$ \\
Control & 6 & 0.0 & $(0.0$, & $1.0)$ & 0.0 & $(0.0$, & $1.0)$ & 0.004 \\
Total MDQ Score & 46 & 22.0 & $(12.0$, & $41.0)$ & 34.0 & $(17.0$, & $52.5)$ & $<0.001$ \\
\hline
\end{tabular}

Data are presented as medians (25th percentile, 75 th percentile). ${ }^{*}$ Wilcoxon signed rank test was used.

\subsection{Symptoms of Eating Habit Before and During Menstruation}

The prevalence rate of the symptoms of eating habits before menstruation was increased appetite $(70.2 \%)$, craving for sweets (73.0\%) and craving for snacks (51.1\%). The rate during menstruation was increased appetite (56.7\%), craving for sweets $(71.6 \%)$ and craving for snacks $(46.1 \%)$. All of the items had a higher prevalence before menstruation than during menstruation. The intensity of each symptom was significantly higher before menstruation than during menstruation (Table 5).

Table 5. Prevalence of eating habit symptoms before and during menstruation by the level of intensity and comparison of eating habit symptoms intensity before and during menstruation $(\mathrm{n}=141)$

\begin{tabular}{|c|c|c|c|c|c|c|c|c|c|c|c|c|c|}
\hline \multirow{3}{*}{$\begin{array}{l}\text { Menstrual } \\
\text { phase }\end{array}$} & \multirow{3}{*}{ Symptoms } & \multicolumn{10}{|c|}{ Intensity } & \multirow{3}{*}{$\begin{array}{l}\text { Median } \\
\text { (25th percentile, } \\
\text { 75th percentile) }\end{array}$} & \multirow{3}{*}{$p$-value ${ }^{*}$} \\
\hline & & \multicolumn{2}{|c|}{ None } & \multicolumn{2}{|c|}{ Mild } & \multicolumn{2}{|c|}{ Moderate } & \multicolumn{2}{|c|}{ Strong } & \multicolumn{2}{|c|}{ Severe } & & \\
\hline & & $\mathrm{n}$ & $\%$ & $\mathrm{n}$ & $\%$ & $\mathrm{n}$ & $\%$ & $\mathrm{n}$ & $\%$ & $\mathrm{n}$ & $\%$ & & \\
\hline \multirow{4}{*}{ Before } & $\begin{array}{l}\text { Increased } \\
\text { appetite }\end{array}$ & 42 & 29.8 & 21 & 14.9 & 17 & 12.1 & 40 & 28.4 & 21 & 14.9 & $\begin{array}{l}2.0 \\
(0.0,3.0)\end{array}$ & \multirow{2}{*}{$\begin{array}{l}\text { Before vs during } \\
\text { Increased } \\
\text { appetite } \\
(\mathrm{p}<0.001)\end{array}$} \\
\hline & $\begin{array}{l}\text { Craving for } \\
\text { sweets }\end{array}$ & 38 & 27.0 & 27 & 19.1 & 24 & 17.0 & 32 & 22.7 & 20 & 14.2 & $\begin{array}{l}2.0 \\
(0.0,3.0)\end{array}$ & \\
\hline & $\begin{array}{l}\text { Craving for } \\
\text { snacks }\end{array}$ & 69 & 48.9 & 26 & 18.4 & 24 & 17.0 & 10 & 7.1 & 12 & 8.5 & $\begin{array}{l}1.0 \\
(0.0,2.0)\end{array}$ & \multirow{2}{*}{$\begin{array}{l}\text { Before vs during } \\
\text {-Craving for } \\
\text { sweets }(\mathrm{p}=0.029)\end{array}$} \\
\hline & $\begin{array}{l}\text { Increased } \\
\text { appetite }\end{array}$ & 61 & 43.3 & 20 & 14.2 & 32 & 22.7 & 22 & 15.6 & 6 & 4.3 & $\begin{array}{l}1.0 \\
(0.0,2.0)\end{array}$ & \\
\hline \multirow[t]{2}{*}{ During } & $\begin{array}{l}\text { Craving for } \\
\text { sweets }\end{array}$ & 40 & 28.4 & 32 & 22.7 & 30 & 21.3 & 28 & 19.9 & 11 & 7.8 & $\begin{array}{l}1.0 \\
(0.0,3.0)\end{array}$ & \multirow{2}{*}{$\begin{array}{l}\text { Before vs during } \\
\text { Craving for } \\
\text { snacks }(\mathrm{p}<0.001)\end{array}$} \\
\hline & $\begin{array}{l}\text { Craving for } \\
\text { snacks }\end{array}$ & 76 & 53.9 & 28 & 19.9 & 24 & 17.0 & 5 & 3.5 & 8 & 5.7 & $\begin{array}{l}0.0 \\
(0.0,2.0)\end{array}$ & \\
\hline
\end{tabular}

"Wilcoxon signed rank test was used to compare the median of intensity of symptoms before and during menstruation.

\section{Discussion}

This study was conducted from May to July 2021, more than a year into the COVID-19 pandemic, thus it may affect a little to the survey results of the menstruation-related symptoms of university students in Japan. In our study, the prevalence rates of students who had experienced at least one symptom listed on the MDQ 46 items 
before and during menstruation were $96.5 \%$ and $99.3 \%$, respectively. The prevalence rate before menstruation was similar to the results of a previous study which used the MDQ 46 items (Matsumoto, Egawa, Kimura, \& Hayashi, 2019). However, the prevalence rate during menstruation was higher than the result of a meta-analysis of prevalence for young women at 71.1\% (Armour et al., 2019). Most of the students in the present study had symptoms during menstruation, but the number of symptoms or intensity of symptoms varied greatly depending on the individual. Some students may be diagnosed as PMS or dysmenorrhea.

Regarding intensity of the MDQ symptoms, it has been reported that among high school students, the MDQ total score and the scores of pain, water retention, impaired concentration, behavior change, autonomic reaction, and negative effect were lower in the premenstrual phase than in the menstrual phase (Otsuka-Ono, Sato, Ikeda, \& Kamibeppu, 2015). Other studies have shown similar results for university students, as the MDQ total score and the scores of pain, autonomic reaction, impaired concentration, behavior change were lower in the premenstrual phase than in the menstrual phase (Ishihara, 2019; Uemura, Sakae, \& Matsumura, 2014), but scores of negative affect and water retention were not different between the two phases (Ishihara, 2019). Our results were similar to a previous study conducted before the COVID-19 pandemic, which reported that the intensity of pain, autonomic reaction, impaired concentration, behavior change and MDQ total symptoms for young women may be stronger during menstruation than before menstruation.

We found that the ten most frequently experienced symptoms before menstruation were skin blemish or disorder, mood swings, irritability, swelling, cramps, fatigue, take naps, stay in bed, feeling sad or blue, weight gain and difficulty concentrating, which were most agreed with previous study (Matsumoto et al., 2019). Skin blemish, which was the most prevalent symptom in our study, may occur when sebum secretion increases due to increased progesterone secretion after ovulation. However, the prevalence rate of the skin disorder for university students varies greatly from $36 \%$ in Iran (Ghiasi, Keramat, \& Mollaahmadi, 2018) to $80 \%$ for acne in Palestine (Abu Alwafa, Badrasawi, \& Haj Hamad, 2021). Moreover, 56\% of adults in USA reported that their acne got worse before menstruation (Geller, Rosen, Frankel, \& Goldenberg, 2014), and 44\% of adult women experienced premenstrual flares of their acne but this was not affected by ethnicity (Stoll et al., 2001). Thus, the difference in prevalence seems to depend on country-specific factors.

In our study, cramps, take naps, stay in bed, feeling sad or blue and difficulty concentrating were also common symptoms in the premenstrual phase. On the other hand, the cramps, take naps, stay in bed, feeling sad or blue, difficulty concentrating were not contained in the top ten of the most frequently experienced symptoms using the MDQ in previous research (Matsumoto et al., 2019). There was a significant increase in reported mental health symptoms including low mood, poor sleep, lowliness, poor concentration since the outbreak of the pandemic and the women who had these symptoms were more likely to have painful periods and worsening premenstrual symptom (Phelan et al., 2021). Therefore, according to our research results, the symptoms of lower abdominal pain, dozing, and difficulty concentrating may occur due to the worsening mental state caused by COVID-19.

Regarding symptoms during menstruation, previous studies mainly focused on the menstruation pain or dysmenorrhea, and there are less studies focusing on other menstruation related symptoms. We clarified the prevalence and intensity of the symptoms using MDQ and three eating items. Our results are consistent with previous studies in showing that the prevalence rate of cramps was as high as 79.2\% (Yamamoto, Okazaki, Sakamoto, \& Funatsu, 2009) and 86.5\% (Ghiasi et al., 2018). Similarly, fatigue was reported as common as $84.5 \%$ (Ghiasi et al., 2018). Other studies also have reported prevalence rates of some other symptoms such as mood swings (71.8\%), depression (62.7\%) (Ghiasi et al., 2018) and irritability (76.3\%) (Fernández-Martínez, Onieva-Zafra, Abreu-Sánchez, Fernández-Muñóz, \& Parra-Fernández, 2019). Our results seem to show higher prevalence rates of mood swings and depression than the above studies. In a study conducted after the COVID-19 pandemic, 30\% had new dysmenorrhea and increased psychological symptoms such as low mood, anxiety, lowliness during pandemic (Phelan et al., 2021). Because students have had to stay at home during the pandemic and cannot often communicate with others, this may influence the negative affect items, such as mood swings and depression.

Before the COVID-19 pandemic, we showed that the rate of increased consumption of sweets and snack in young students was $68.4 \%$ and $40 \%$, respectively, before menstruation, and $47.9 \%$ and $23 \%$, respectively, during menstruation (Matsuura et al., 2020). In the present study, which was conducted during the pandemic, we showed that the prevalence rate of the increased appetite and craving for sweets before menstruation was higher than the MDQ 46 symptoms, and that the rate of the craving for sweets during menstruation was the fifth most prevalent symptom. A study in UAE showed that $71 \%$ of university students had a craving for sweets and $72 \%$ had increased appetite (Hashim et al., 2019). Moreover, the marked change in appetite, over-eating, or specific food craving is 
one of the diagnoses of PMDD (American Psychiatric Association, 2013), and these changing eating habits are common in the premenstrual phase. In our previous study, we also found that increased appetite and intake of sweets are associated with depression, anger and sleepiness before menstruation, while intake of junk food such as snacks is associated with irritability and depression (Matsuura et al., 2020). These symptoms of eating behavior may be associated with psychological symptoms caused by COVID-19 pandemic.

The premenstrual symptoms and dysmenorrhea are reported to affect school life and academic performance (Armour et al., 2019; Tadakawa et al., 2016). Recently, it has been reported that dysmenorrhea increases the risk of hypertensive disorders of pregnancy (Nakayama et al., 2020) and increases the risk of giving birth to a low-birth-weight baby (Feng et al., 2021), while PMS increases the risk of hypertension (Bertone-Johnson, Whitcomb, Rich-Edwards, Hankinson, \& Manson, 2015). Thus, dysmenorrhea and PMS may influence the future health of young women. It implies that we should advise young students to visit the medical doctors in order to receive appropriate treatments.

This study has several limitations. First, the participants were limited to a department in a university and the sample size was small, which makes generalization of these results difficult. Second, the participants may have recall bias in describing their own experiences of menstruation symptoms. Third, the causal relationships with COVID -19 pandemic and menstruation related symptoms cannot be evaluated since this was a cross-sectional survey. A longitudinal study may be needed to study the changes of menstruation-related symptoms by using MDQ in other areas or in participants with different backgrounds.

\section{Conclusion}

In this study, which was conducted during the time of the COVID-19 pandemic, using the MDQ we found that scores of pain, autonomic reaction, impaired concentration, behavior change, and control were significantly higher during the menstruation phase compared to the scores of pre-menstruation phase. It may suggest that the exposure to the COVID-19 pandemic may affect more psychological symptoms rather than the physical symptoms.

\section{Acknowledgments}

This work was supported by JSPS KAKENHI Grant Number JP20K10830. The authors are deeply grateful to the participants.

\section{Competing Interests Statement}

The authors declare that there are no competing conflicts of interest.

\section{References}

Abu Alwafa, R., Badrasawi, M., \& Haj Hamad, R. (2021). Prevalence of premenstrual syndrome and its association with psychosocial and lifestyle variables: a cross-sectional study from Palestine. BMC Women's Health, 21(1), 233. https://doi.org/10.1186/S12905-021-01374-6

American Psychiatric Association. (2013). Diagnostic and Statistical Manual of Mental Disorders (5th ed.). Arlington, VA: American Psychiatric Publishing.

Armour, M., Parry, K., Manohar, N., Holmes, K., Ferfolja, T., Curry, C., ... Smith, C. A. (2019). The Prevalence and Academic Impact of Dysmenorrhea in 21,573 Young Women: A Systematic Review and Meta-Analysis. Journal of Women's Health, 28(8), 1161-1171. https://doi.org/10.1089/jwh.2018.7615

Bertone-Johnson, E. R., Whitcomb, B. W., Rich-Edwards, J. W., Hankinson, S. E., \& Manson, J. A. E. (2015). Premenstrual Syndrome and Subsequent Risk of Hypertension in a Prospective Study. American Journal of Epidemiology, 182(12), 1000-1009. https://doi.org/10.1093/aje/kwv159

Clayton, A. H. (2008). Symptoms related to the menstrual cycle: diagnosis, prevalence, and treatment. Journal of Psychiatric Practice, 14(1), 13-21. https://doi.org/10.1097/01.pra.0000308491.54885.f8

Demir, O., Sal, H., \& Comba, C. (2021). Triangle of COVID, anxiety and menstrual cycle. Journal of Obstetrics and Gynaecology, 41(8), 1257-1261. https://doi.org/10.1080/01443615.2021.1907562

Feng, B., Li, H., Peng, Y., Jiang, Q., Wu, Y., Liu, S., ... Huang, D. (2021). A cohort study of dysmenorrhea and risk of low birth weight. The Journal of Maternal-Fetal \& Neonatal Medicine, 1-7. https://doi.org/10.1080/14767058.2021.1914581

Fernández-Martínez, E., Onieva-Zafra, M. D., Abreu-Sánchez, A., Fernández-Muñóz, J. J., \& Parra-Fernández, M. L. (2019). Absenteeism during Menstruation among Nursing Students in Spain. International Journal of Environmental Research and Public Health, 17(1), 53. https://doi.org/10.3390/ijerph17010053 
Freitas, F. da F., de Medeiros, A. C. Q., \& Lopes, F. de A. (2021). Effects of Social Distancing During the COVID-19 Pandemic on Anxiety and Eating Behavior-A Longitudinal Study. Frontiers in Psychology, 12, 645754. https://doi.org/10.3389/fpsyg.2021.645754

Geller, L., Rosen, J., Frankel, A., \& Goldenberg, G. (2014). Perimenstrual Flare of Adult Acne. The Journal of Clinical and Aesthetic Dermatology, 7(8), 30-34. Retrieved from /pmc/articles/PMC4142818/

Ghiasi, A., Keramat, A., \& Mollaahmadi, L. (2018). The relationship between attitudes toward menstruation and perimenstrual symptoms among female students of shahroud university of medical sciences, northeast Iran. Shiraz E Medical Journal, 19(8), e65714. https://doi.org/10.5812/semj.65714

Hashim, M. S., Obaideen, A. A., Jahrami, H. A., Radwan, H., Hamad, H. J., Owais, A. A., ... Faris, M. A. I. E. (2019). Premenstrual Syndrome Is Associated with Dietary and Lifestyle Behaviors among University Students: A Cross-Sectional Study from Sharjah, UAE. Nutrients, 11(8), 1939. https://doi.org/10.3390/nu11081939

Ishihara, F. (2019). Stress Assessment of Young Women in Each Menstrual Cycle Phase. Bulletin of the Osaka Jonan Women's Junior College, 53, 75-90.

Jalal, S. M., Beth, M. R. M., Al-Hassan, H. J. M., \& Alshealah, N. M. J. (2021). Body Mass Index, Practice of Physical Activity and Lifestyle of Students During COVID-19 Lockdown. Journal of Multidisciplinary Healthcare, 14, 1901-1910. https://doi.org/10.2147/JMDH.S325269

Matsumoto, T., Egawa, M., Kimura, T., \& Hayashi, T. (2019). A potential relation between premenstrual symptoms and subjective perception of health and stress among college students: a cross-sectional study. BioPsychoSocial Medicine, 13, 26. https://doi.org/10.1186/s13030-019-0167-y

Matsuura, Y., Inoue, A., Kidani, M., \& Yasui, T. (2020). Change in appetite and food craving during menstrual cycle in young students. International Journal of Nutrition and Metabolism, 12(2), 25-30. https://doi.org/10.5897/IJNAM2019.0264

Moos, R. H. (1968). The development of a menstrual distress questionnaire. Psychosomatic Medicine, 30(6), 853-867. https://doi.org/10.1097/00006842-196811000-00006

Moos, R. H. (2010). Menstrual distress questionnaire manual, instrument, and scoring guide ( $4^{\text {th }}$ ed.). Menlo Park, CA: Mind Garden, Inc.

Nakayama, M., Ono, M., Iizuka, T., Kagami, K., Fujiwara, T., Sekizuka-Kagami, N., ... Fujiwara, H. (2020). Hypertensive disorders of pregnancy are associated with dysmenorrhea in early adulthood: A cohort study. Journal of Obstetrics and Gynaecology Research, 46(11), 2292-2297. https://doi.org/10.1111/jog.14431

Otsuka-Ono, H., Sato, I., Ikeda, M., \& Kamibeppu, K. (2015). Premenstrual Distress Among Japanese High School Students: Self-Care Strategies and Associated Physical and Psychosocial Factors. Women \& Health, 55(8), 859-882. https://doi.org/10.1080/03630242.2015.1061089

Perez-Dominguez, F., Polanco-Ilabaca, F., Pinto-Toledo, F., Michaeli, D., Achiardi, J., Santana, V., ... Rebolledo, C. (2021). Lifestyle Changes Among Medical Students During COVID-19 Pandemic: A Multicenter Study Across Nine Countries. Health Education \& Behavior, 48(4), 446-454. https://doi.org/10.1177/10901981211019292

Phelan, N., Behan, L. A., \& Owens, L. (2021). The Impact of the COVID-19 Pandemic on Women's Reproductive Health. Frontiers in Endocrinology, 12, 642755. https://doi.org/10.3389/fendo.2021.642755

Stoll, S., Shalita, A. R., Webster, G. F., Kaplan, R., Danesh, S., \& Penstein, A. (2001). The effect of the menstrual cycle on acne. Journal of the American Academy of Dermatology, 45(6), 957-960. https://doi.org/10.1067/mjd.2001.117382

Tadakawa, M., Takeda, T., Monma, Y., Koga, S., \& Yaegashi, N. (2016). The prevalence and risk factors of school absenteeism due to premenstrual disorders in Japanese high school students-a school-based cross-sectional study. BioPsychoSocial Medicine, 10, 13. https://doi.org/10.1186/S13030-016-0067-3

Takmaz, T., Gundogmus, I., Okten, S. B., \& Gunduz, A. (2021). The impact of COVID-19-related mental health issues on menstrual cycle characteristics of female healthcare providers. Journal of Obstetrics and Gynaecology Research, 47(9), 3241-3249. https://doi.org/10.1111/jog.14900

Uemura, Y., Sakae, R., \& Matsumura, K. (2014). Relationship between self-management in menstruation and menstrual symptoms. Japanese Journal of Maternal Health, 54(4), 512-518. Retrieved from 
https://ci.nii.ac.jp/naid/110009688137

Yamamoto, K., Okazaki, A., Sakamoto, Y., \& Funatsu, M. (2009). The relationship between premenstrual symptoms, menstrual pain, irregular menstrual cycles, and psychosocial stress among Japanese college students. Journal of Physiological Anthropology, 28(3), 129-136. https://doi.org/10.2114/jpa2.28.129

\section{Copyrights}

Copyright for this article is retained by the author(s), with first publication rights granted to the journal.

This is an open-access article distributed under the terms and conditions of the Creative Commons Attribution license (http://creativecommons.org/licenses/by/4.0/). 